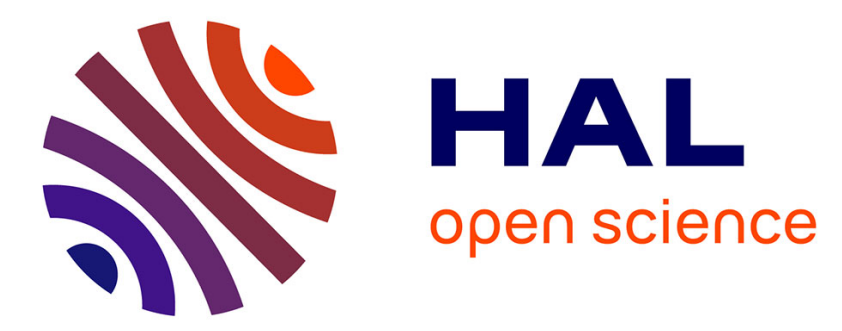

\title{
Recherches sur le développement urbain sous l'Empire romain dans le Nord de la France \\ Ernest Will
}

\section{To cite this version:}

Ernest Will. Recherches sur le développement urbain sous l'Empire romain dans le Nord de la France. Gallia - Fouilles et monuments archéologiques en France métropolitaine, 1962, 20 (1), pp.79-101. 10.3406/galia.1962.2349 . hal-01926427

\section{HAL Id: hal-01926427 \\ https://hal.science/hal-01926427}

Submitted on 25 Feb 2020

HAL is a multi-disciplinary open access archive for the deposit and dissemination of scientific research documents, whether they are published or not. The documents may come from teaching and research institutions in France or abroad, or from public or private research centers.
L'archive ouverte pluridisciplinaire HAL, est destinée au dépôt et à la diffusion de documents scientifiques de niveau recherche, publiés ou non, émanant des établissements d'enseignement et de recherche français ou étrangers, des laboratoires publics ou privés.

\section{(ㅇ)(1) $\$$}

Distributed under a Creative Commons Attribution - NonCommercial - NoDerivatives| 4.0 


\title{
RECHERCHES SUR LE DÉVELOPPEMENT URBAIN SOUS L'EMPIRE ROMAIN DANS LE NORD DE LA FRANCE
}

\author{
par Ernest WILL
}

Il y a une trentaine d'années paraissait comme "contribution à l'histoire urbaine du Nord de la France", de la plume d'un savant belge, F. Vercauteren, une Étude sur les civitates de la Belgique Seconde1. Cet ouvrage reste toujours "valable", comme on dit. pour l'essenticl et il ne peut guère en être autrement. Les sources littéraires ou épigraphiques n'ont pas connu d'enrichissement notable depuis cette date et si la discussion de ces documents n'est pas close, elle n'est pas susceptible non plus d'apporter des changements considérables. Le problème du statut juridique des agglomérations urbaines et celui des incidences de ce statut sur leur développement en restent exactement où ils en étaient depuis longtemps et il est clair, par ailleurs, que si les conditions économiques ou autres s'y prêtaient, une agglomération quelconque pouvait prendre des caractères urbains, comme il est arrivé aussi en d'autres temps. Les seules données nouvelles dans notre connaissance du problème sont fournies par les découvertes archéologiques. La guerre et ses conséquences ainsi que le développement général des fouilles en tous lieux ont apporté aussi pour cette zone de la Belgique Seconde des informations fraîches; et s'il est vrai que ces informations restent toujours fragmentaires - elles n'intéressent qu'un petit nombre des cités envisagées .... il ne sera pas sans intérêt d'en tirer parti dès aujourd'hui comme contribution à l'histoire urbaine du Nord de la France et - à la diférence de ce qui fut le programme de F. Vercauteren - pour toute la période de l'Antiquité et surtout celle du IIaut-Empire. Les précisions obtenues ailleurs, en Allemagne, en Suisse, en Grande-Bretagne permettront d'étayer plus solidement les conclusions qui semblent se dessiner. Mais pour arriver à ces conclusions nous envisagerons successivement quelques cas particuliers.

\section{Amiens-SAMAROBRIVA.}

Les découvertes archéologiques nous ont fourni, au bout de quinze années d'observations minutieuses, une image d'une précision inattendue et, en définitive, admirable, de la ou des conceptions urbanistiques qui ont présidé à la naissance d'Amiens-Samarobriva et qui ont conditionné son histoire pendant les quatre siècles d'Empire romain et au-delà.

(1) Paru dans les .Mémoires de l'Acad. Belge, Lellres, t. 33 (1934); le sous-titre précise la date : " de la fin du III ${ }^{\mathrm{e}}$ à la fin du $x{ }^{e}$ siècle $n$. Cf. plus récemment, S. J. de LAFr, Schets van het ontslaan en de ontwikkeling van stedelijke Agglomeratie in Noord-Gallië, dans Meded. k. Vlam. Acad. Welens. Letleren, 22, 1960, 6, exposé général sur les conditions politiques, juridiques et économiques du développement urbain, dans lequel l'archéologie tient peu de place. 
Comme la ville artuelle ne conserve plus le sourenir visible d'aucun monument antique et, que ces monuments, qui ailleurs font encore la parure des sites modernes, nous restent médiocrement connus ... on possède des données sur deux d'entre rux seulement, lamphithéatre et un batiment de thermes -, il convient d'autant plus de souligner que le plan d'ensemble de la ville antique nous apparait avere une netteté que nous ne retrouvons ni dans le voisinage immédiat, ni à des distances considérables a la ronde : on ne saurait, en cffet, ciler de parallèle ni dans la moitié nord de la France ni en Belgrique. Comme j’ai cu l'oceasion d'exposer ailleurs, en collaboration aver le fouilleur, M. F. Vasselle, le détail des recherches et des domnés, il me suffira ici de rappeder les eléments essentiels de nos ronnaissancess 2 .

La base est foumir par ce que nous savons aujourd'hui sur le réseau des voies antiques dans la ville; en une cinquantaine de points. en effet. la présence de cess voies a pu ètre constatée et leur orientation notée. Ine observation toute récente - - concernant la voie Cord-Surl no III, a l'Est des thermes - vient corroborer, si besoin etait, le dessin fourni il y a deux ans. Mais voici les résultats.

Nous sommes en présener d'un plan à damier parfaitement régulier (fig. 1); quand nous rencontrons des voies en oblique par rapport a ce damier. nous pourons leur faire sans peine une place dans l'étal correspondant au Bas-limpire. Ce damier est dans son orientation d'une uniformité et d'une régularité plus grandes que celles notées parfois ailleurs et il est ainsi d'autant plus frappant que les rectangles qui forment le quadrillage rhangent de dimensions, mais de facon telle que l'on distingue très nettement deux zones : un noyau plus pelit qui groupe les rectangles plus petits (1:5) $\times 160$ mètres) localisés dans l'angle nord-est du plan, et une zone plus étendue. entourant le noyau précédent et groupant les rectangles plus grands $(160 \times 160$ mètres). Tout se passe, à première vue, déja, comme si un plan primitif avait connu un agrandissement à une certaine date. Or deux observations viennent confirmer cette vue. Il se trouve, en effet, que l'amphithéattre est situé en pleine ville dans le damier définitif ; la chose est anormale, on le sait, puisque, ronformément à une règle assez bien établie, ces monuments s'élevaient en bordure des zones habitées ( $\cdot f$. un bon exemple à Vetera-Xanten, dont le plan n'a pas varié). Mais tout s'explique quand on constate que l'amphithéâtre se troure situé très exactement sur la bordure ouest du plan primitif et encore en dehors de la zone à petits rectangles et que, d'ailleurs, sa présence a manifestement causé quelque trouble dans le dessin régulier des rertangles de dimensions plus grandes : on en conclura que la zone des premiers représente Ie plan primitif. I) plus, le batiment des thermes qui. lui, oceupe a peu près en entier un des grands reclangles, un peu au Sud de l'amphithéatre, recouvrait les vestiges d'une construction antérieure ol a révélé dans ses fondations des restes sculptés de monuments divers, funéraires ou autres ${ }^{3}$ évidemment plus anciens : ce sont la deux indices de remanie-

(2) Trois etudes récentes utilisent les donnces que les fouilles ont fournies sur l'histoire d'Amiens : F.VAsslit.t.EE. Wur., Ies cimelieres gallo-romains d'Amiens, Rev. Nord, 1956, pp. 321-330; L'enceinte du Bas-Lmpire el l'histoire de la ville d'Amiens, ibid., 1958, pp. 467-482; Lurbanisme romain à Samarubrima-4miens, ibid., 1960, pp. 327-353. C'est dans cette derniere etude que l'on trouvera l'analỵse du plan à damier ; pour un plan plus détaillé du centre de la ville, ef. Rer. Nird, 1958, pp. 476-477.

(3) Cf. Rer. Nord, 1960, pp. 341-312. Pour les fragments d'architecture el de sculpture découverts dans le's 


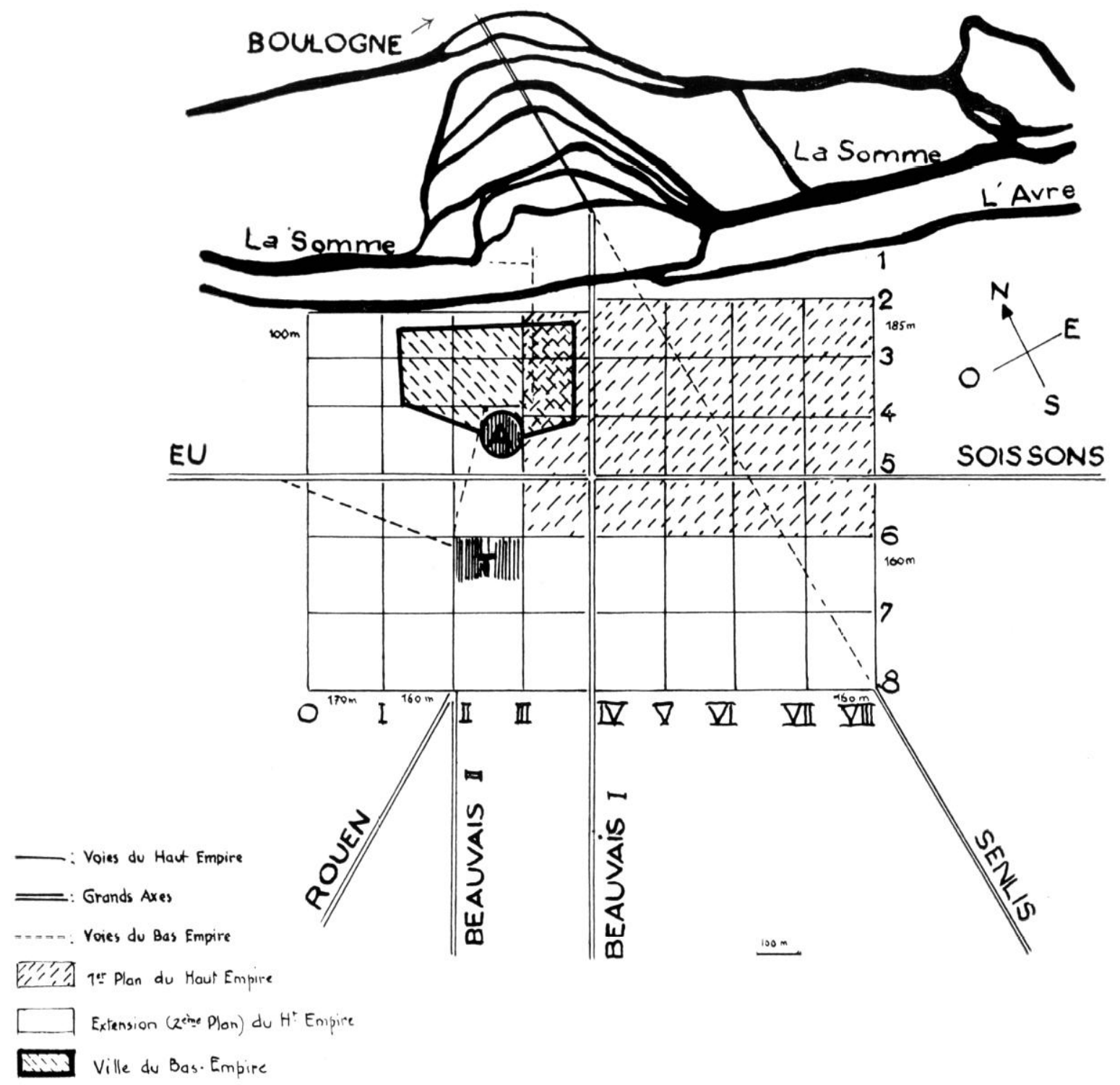

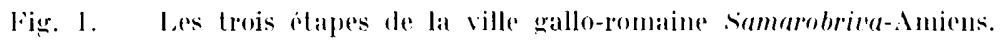

ments considérables et précisément les thermes, dont l'édification doit se placer au ne siecle, sont situés cux aussi en bordure du plan à petits rectangles. L'hypothèse de l'élargissement. d'un plan primitif semble donc amplement confirmée. et nous pouvons résumer ainsi les résultats auxquels nous avons abouti.

1. Plan primitif: une surface totale de 40 hectares se décomposant ainsi :

5 rangées d'insulae dans le sens Est-Ouest, ou $160 \times 5=800$ mètres;

4 rangées d'insulae dans le sens Yord-sud, ou $125 \times 4=500$ mètres.

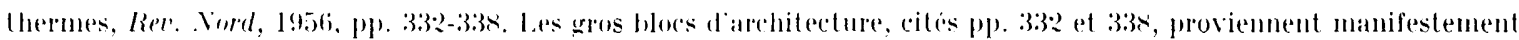
dun grand momumest pullic porte an are de triomphe?" : la prisence de vestiges de monuments funcraires pent confirmer yuon se trouvait a cette place sur les limites de la ville du premier plan. 
II. Plan définitif: une surface lolale d'environ 10:5 hectares se décomposant ainsi :

8 rangées d'insulae dans le sens Ouest-Wst, ou $7 \times 160+1 \times 170=1290$ mètres ;

6 rangées d'insulae dans le sens Nord-sud, ou $125 \times 4+160 \times 2=820$ mètres.

Ces chiffres demandent quelque commentaire. Pour ce qui est de la surface lotale, nous y reviendrons dans nos conclusions et il nous suflira de dire pour l'instant que Samarobriva fait figure de grande ville dans la région. Quelques remarques s'imposent cependant ici sur les dimensions que nous avons reconnues aux insulae. Il s'agit, dans une cerlaine mesure du moins, d'un chiffre théorique. Il est, en effet, infiniment rare que l'on ait pu établir les dimensions totales des rues. leur largeur complète en particulier avec fossés et trottoirs; il semble toutefois que dans leurs dimensions mêmes ainsi que dans leur structure les rues d'Amiens gallo-romain ressemblaient beaucoup à celles dont l'image a pu ètre restituée ailleurs ${ }^{4}$. I)ans la pratique, ce qu'on peut établir avec une bonne approximation. c'est l'espacement des voies, en quelque sorte d'axe en axe, et les chiffres ainsi obtenus semblent tolérer une interprétation satisfaisante. Ainsi à l'entraxe de 160 mètres peut correspondre une insula de 100 pas romains de còté, ou $147 \mathrm{~m}$. 50, ce qui laisse $12 \mathrm{~m}$. 50 pour la chaussée ; pour l'entraxe de 125 mètres. on songerait volontiers au chifire de 80 pas romains ou 118 mètres mais il resterait une dimension apparemment trop faible pour la voie, el il faut préférer peut-ètre 75 pas ou $110 \mathrm{~m} .60$.

L'importance absolue de ces chillres peut surprendre : les insulae de Samarobriva possédaient des dimensions notables. Il n'est malheureusement pas des plus aisés de réunir des termes de comparaison. Rien dans le voisinagre immédiat. en France en particulier. Des données très précises sont signalées à Augusta Raurica en Suisse avec les chifires de $\overline{5} \overline{5} \times 66$ mètres pour l'insula normale. $\Lambda$ Trèves et à Cologne cependant, on semble at teindre une centaine de mètres pour le còté des insulae. Mais des chifires comparables proviennent surtout de la Bretagne romaine : 100, 120 ou 125 mètres apparaissent à Verulamium, à Inurovernum-Canterbury et à Calleva-Silchester ${ }^{5}$. Nos connaissances d'ensemble sur ce problème restent cependant trop insuffisantes pour qu'on puisse faire plus que fournir ces données.

Nous ne saurions quitter le plan en damier de Samarobriva sans nous attarder un instant à une: question de méthode. Avant les résultats des fouilles que nous venons d'exposer, on ne pouvait se faire qu'une idée très vague du plan des villes de la Gaule du Nord - idée qui reposait sur la généralisation de constatations de détails faites avec plus ou moins de bonheur en d'autres régions. Amiens même nous offre un cas curieux des erreurs qui pouvaient résulter de généralisations de ce genre.

On en était arrivé, en effet, à localiser la ville du Bas-Empire autour de la cathédrale et parmi les arguments qu'on croyait devoir mettre en avant - . on peut relire l'argumentation résuméc dans l'ouvrage de F. Vercauteren cité en lête de cet articlé 6 ... figurait

(4) Nous avons publié dans Kev. Nord, 1960 une série de coupes pratiquées sur les voies romaines d'Amiens. I a plus complète, p. 344, fig. 2, ne fournit pas de renseignements suflisants sur les bas-còtés de la chaussée. On rapprochera les remarques de R. Laur-Belart, sur les rues d'Augst, dans Führer durch Augusla Raurica, pp. 26-27 el fig. 6, qui révèlent un état très proche de celui d'Amiens.

(5) Aucune étude récente ne semble avoir été consacrée à ce problème du quadrillage. Cif. pour les endroits cités (bibl. sommaire) : Cologne, P. I. В BLwE, Colonia Agrippinensis, fig. 57 ; Н. Schмrт, Sladt und Imperium, Köln in röm. Zeil, p. 93, fig. 7 ; Trives, (inswen, Manuel, III, 1, p. 258, fig. 68; v. . Mssow, Trierer Zeilschr., 1956, p. 77; Augusia Raurica, R. Lavr-Bristat, Führer, p. 24 et plan d'ensemble en h. t. Pour Verulamium, ex. gr. Pat i.Y-Wissowa, Realenc. s. v. Verolamium (I. Richmond) ; Canterbury, Sheppard Frere, Roman Canlerbury, fig. 5; C. BooN, Roman Silchesler, plan h. t. - La discussion sur ce qui est cardo et decumanus dans ces villes est loin d'être close et reste souvent confuse. Pour le plan primitif d'Amiens, nous avons été amené à admettre un cours excentrigue pour ces deux artères. Cf. p. ex. pour Cologne, H. v. PETrikovirs, IJas röm. Rheinland, p. 87.

(6) F. Vercattires, Elude sur les civilates, p. 293 et p. 317 (plan). 
l'" aspect rectangulaire " de l'enceinte présumée et "la régularité ", déclarée "assè remarquable ", du tracé des rues. (Ce qui revenait à dire que le tracé des rues modernes avait conservé celui de l'Antiquité et cette correspondance jointe à la proximité de la cathédrale fournissait la preuve majeure. Les fouilles récentes ont fait table rase de cette théorie; la ville du Bas-Empire s'est rérélée dans une toute autre zone, plus à l'Ouest, centrie autour de l'amphithéâtre transformé en "castillon "?. Parmi les rues médiévales ct modernes qui, à l'intérieur de cette enceinte, conservent dans les grandes lignes un tracé antique, une seule suite peut être retenue avcc certitude : rues au lin, des chaudronniers, Saint-Martin, et c'est autour de cette artère que s'est organisée la ville tardive. On sait pour le reste aujourd'hui qu'il n'y a plus lieu pour une date basse de chercher un damier avec cardo et decumanus: on y reviendra à propos de Boulogne. En dehors de l'enceinte, une seule autre artère moderne a conservé avec certitude un tracé antique ; c'est la suite : rues de la République. des Sergents, Flatters et du Bloc. Mais c'est là tout simplement l'antique et (apitale voie venant de Beauvais et filant sur Boulogne; la rue de la ville du llaut-Empire était revenue à l'état de route au Bas-Empire et son tracé s'est encore imposé à la ville du Moyen Age. Mais, pour le reste, dans tout ce qui fut la zone extra muros du Bas-Empire, le quadrillage décrit ci-dessus n'a laissé aucune trace, n'a pu laisser de trace si l'on veut bien se souvenir qu'il n'y avait plus là que terrains vagues correspondant aux ruines du ${ }_{\text {III }} \mathrm{e}$ siècle et retournant lentement à l'état de cultures. La route de Senlis traversait maintenant toute cette zone en diagonale pour rejoindre le passage de la Somme et son tracé, bien reconnu en divers endroits, est la meilleure preuve de la disparition de l'agglomération urbaine sur ses bords à l'époque tardive où elle fut en usage.

Ces faits, solidement établis, mettront en garde contre une interprétation trop rapide et trop systématique des tracés, considérés comme "parlants", des rues médiévales ou modernes. Cie qui est vrai à Amiens l'est certainement non moins ailleurs dans toute cette. vaste région qui connut les ravages profonds des invasions du in ${ }^{\mathrm{e}}$ siècle et qui vit succéder aux grandes villes du Haut-Empire de petites enceintes resserrées. Dans toute cette région, le prétendues "survivances" doivent toujours ètre soigneusement vérifiées et rien ne vaudra dans ce domaine le témoignage archéologique ${ }^{8}$.

Il est regrettable que les données dont nous venons de faire état ne puissent pas s'inscrire plus aisément dans un cadre chronologique. La ville romaine succède à une ville gauloise dont elle conserve le nom : Samarobriva, Pont sur la Samara, la future Somme. C'est vers ce pont que se dirigeaient les deux grandes voies dont nous venons de faire état ci-dessus et que se dirigeail aussi une troisième, et non la moins importante, celle qui venait de Soissons. Quel était l'emplacement de l'agglomération gauloise? Aucune donnée certaine ne nous permet de répondre à cette question et on peut rappeler seulement un indice fugitif : immédiatement au Nord de la cathédrale se trouve le port du Ion et la rue du Don ${ }^{9}$. Si la toponymie était une science moins trompeuse, on reconnaitrait volontiers là un

(7) C.f. notre étude, Rev. Nord, 1958 , pp. 167 suiv.

(8) C'est ainsi qu'on est amené à faire les plus expresses réserves sur deux "analỵses " récentes, appliquées à Senlis et à limoges, oủ le quadrillage ancien apparaîtrait encore dans la ville moderne, cf. Grexier, Manuel, III, 1, p. 2.46 et 250 (essais de (i. Matherat ${ }_{i}$. Le cas de Senlis ne saurait, en réalité, guère différer de celui d'Amiens ou d'autres villes de la région. Notons cependant que le matériel archéologique qui fonde ces analỵses n a pas été publié.

(9) (if. sur le plan publié dans Rev. Nord, 1958, p. 477. 
dunum cellique, le souvenir même de l'ag̣glomération préromaine sans d'ailleurs trop savoir de quel ròté exactement la chercher.

Pour la date de la fondation de la ville romaine, nous ne sommes pas mieux renseignés: elle peut ètre ancienne si l'on tient comple du fail que dans le systeme des voies d'Igrippa Amiens occupait une place notable. Celte fondation toutefois ne s'est pas obligatoirement accompagnese aussitòt de l'élablissement du plan a damier primitif. On allribura simplement re dernier aver une bonne probabilité à un moment quelconque du fer siecle au plus lard ; l'agrandissement révélé par les fouilles doit se placer an ${ }_{1} \mathrm{e}$ siecle el faire partie de l'essor urbain général que connul alors lout l'empire. Pour la fin de ce premier Amiens monumental, nous sommes au contraire mieus renseignés. La ville, au témoignage d'une découverte monétaire $\mathbf{1 0}^{0}$ a dù ètre la viclime du premier assaut des barbares en 2066. l'autres monnaies. des pieces de Probus de 277-278. prises dans la maconnerie du remparl, nous renseignent sur la naissance de la ville fortifiée du Bas-limpire : avere ses 10 heclares de superficie qui nous paraissent marquer une déchéance terrible par rapporl ia la grandeur passée, elle peut encore paraitre splendide à Ammien Marcellin. Cette hourgade mime succombe à un nouvel assaut des harbares en 406. comme nous l'apprend un texte lameux et discute de saint Jéròme. mème si elle n'est pas encore a celle date définitivement "transplantée en (iermanie ".

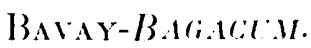

Il n'est pas aisé de se faire une idée exacte de l'extension et du plan de la ville du Haut-Empire à Bavay. Les fouille's régulières se bornent à un seul ensemble monumental ct les découvertes fortuites ne fournissent trop souvent que des informations incomplites ${ }^{11}$.

I'n fait cependant peut ètre considéré comme acquis aujourd'hui : l'aggromération

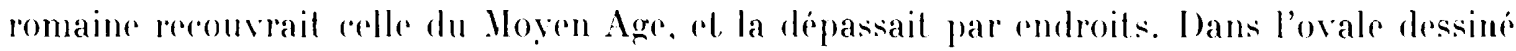
par la levée de terre médiévale (lig. 2), les vestiges romains relevés de façon précise sont de plus en plus nombreux et donnent l'image d'une installation régulière et dense. En nous orientant par rapport au grand ensemble. nous sommes en mesure aujourd'hui de signaler d'abord un blor de maisons au Nord, sur la rue de Valenciennes, des vestiges d'un auter au surd, en bordure de la rue des fiommeries, el une rave a niehes de l'autere coté de la mème rue, dans celle des soupirs. sous l'église mème el les maisons qui lui font face au Nord, re sont les restes d'importants hyporaustes, sans doute ceux des thermes puhlies. C'est ensuite un ensemble assez bien conservé à l'angle sud-est de la (irand'Place, sur la rue des Récollets, ensemble auquel ont correspondu divers vestiges dans la nouvelle perée NordSud partant de la plare. En fin. quand, il y a quelques années, la Grande Rue a été éventré pour la pose d'un rable léléphonique sur la majeure partie de son cours. des restiges d'habitations sont apparus tout au long ; ajoutons que des restes romains ont été signales anciennement is la porte de Mons.

Le probleme est done plutò de savoir de combien la ville antique a pu deborder le radre médieval of quel plan lui fut donné. Qu'elle ait débordé parait assuré par des decouvertes multiples faites au rours des temps (fig. :2). découvertes sur lesquelles nous

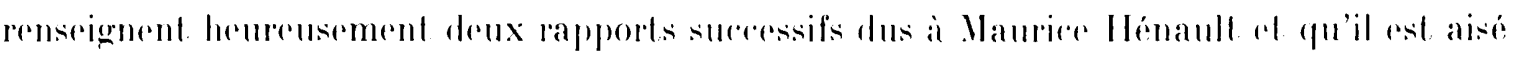

(10) (:f. l. 1., p. 173, ainsi que sur toute lhistoire de la ville du Bas-limpire.

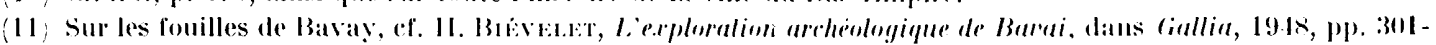

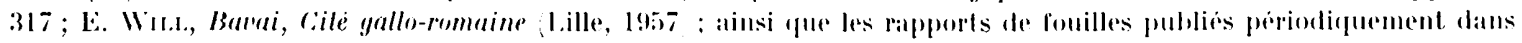
Ciallia; de plus, les itudes de detail cilies ci-apros. 


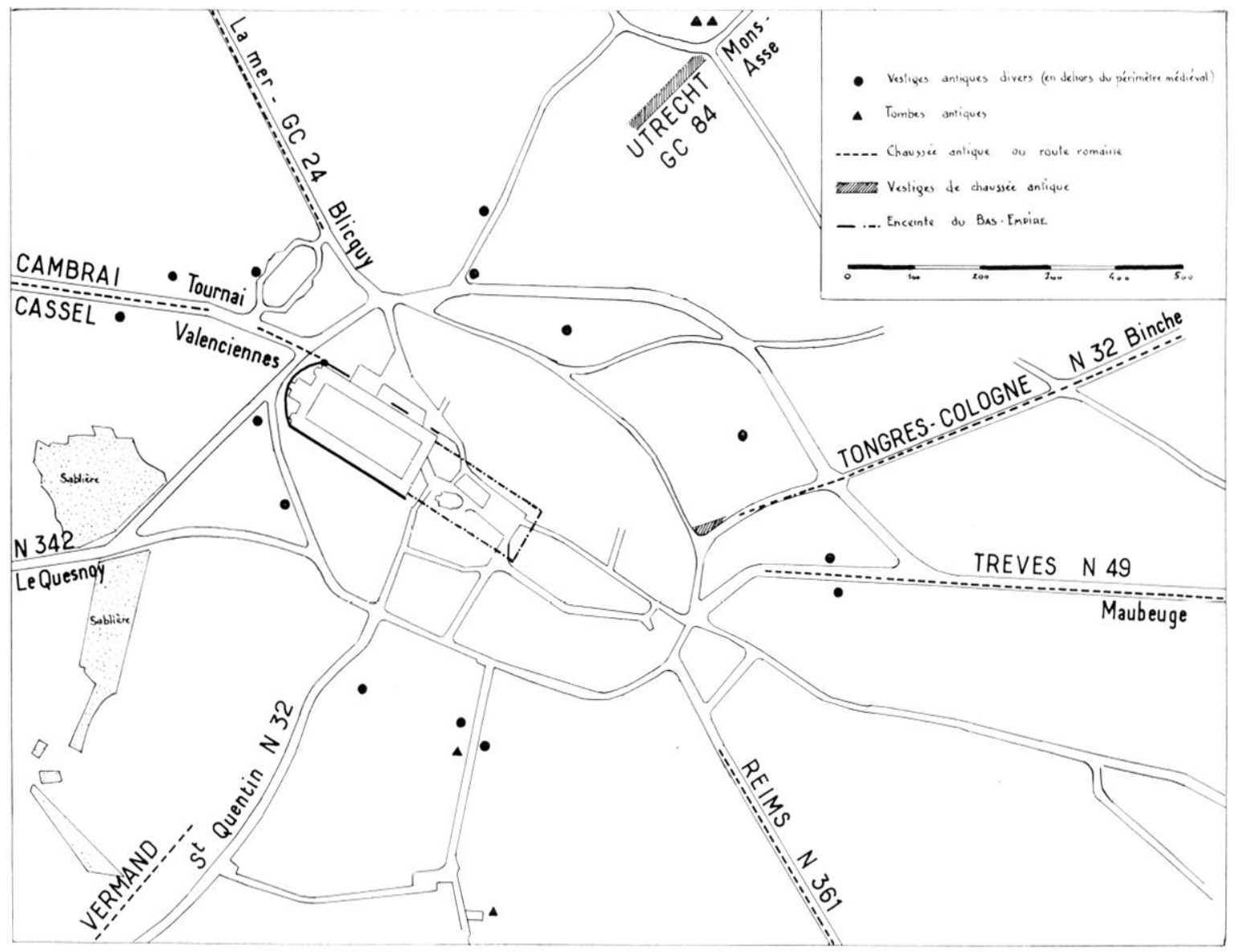

Fig. 2. L.e site de Bagacum-Bavay.

de compléter aujourd'hui ${ }^{12}$. L'enregistrement de res découvertes sur une rarte ne fait naturellement pas apparaître des contours bien nets. Ainsi sur une des grancics sorties, l'actuelle route de Valenciennes, les installations semblent s'étre écrenées assez loin vers l'Ouest; Ir même fait apparait comme probable du còté opposé sur l'actuelle roule de Maubeuge. I)u còté surl-ouest, l'exploitation de toute une série de sablieres nous a domé une image curieuse et confuse à la fois : on semble ètre en présence d'une sorte de faubourge industriel occupé par de nombreux fours à potier, des maisons assez riches (possédant des " caveaux") et enfin un grand nombre de sépultures. I.éturle chronologique de l'abondant. matériel archéologique recueilli dans ce secteur n'a pu ètre assez poussée pour clarifier l'histoire du quartier ; le mot de faubourg semble toutefois convenir assez ${ }^{13}$. Sur les autres còtés. l'existence de cimetières, qui nous sont souvent d'un si grand secours pour une délimitation au moins approximative de la zone habitée, ne nous est guère connue; de façon générale, les tombes n'ont été constatées qu'à une certaine distance de la ville actuelle.

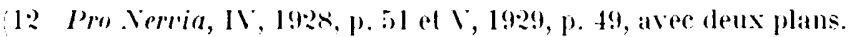

(13) Cf. l'otude de R. Jotsx, l'n quartier arlisanal el residentiel an Sud-Ouesl de Burai, dans Amliquile Gilassique, 1959. 1. $1: 5.5$. 
Sauf sur un point cependant, au Sud, où la petite tombe de Julia Felicula ${ }^{14}$ 'st assez exactement localisée sur les allées de Louvignies; tout auprès. J. Lambiez avait découvert son "temple de Vénus ", c'est-à-dire une cave à niches ornée de stucs peints ${ }^{15}$; nous sommes là manifestement à la charnière des deux villes, celle des vivants et celle des morts. Des constatations faites plus récemment, plus vers l'Ouest, sur la route dr Saint-Quentin, semblent confirmer l'arrêt de l'agglomération à la même hauteur ${ }^{\mathbf{1 6}}$.

Nous obtenons ainsi l'image d'une agglomération principale qui, sur certains côtés. pouvait se prolonger sous la forme de faubourgs. Pour délimiter l'extension de l'agglomération principale, nous aurons recours pour les côtés autres que le côté sud, où la ligne de démarcation nous est apparue déjà, à d'autres indices, fournis surtout par le tracé dés routes. les fameuses routes auxquelles la ville doit son existence. l)u côté de l'Est, en effet. on voit les deux chaussées de Tongres et de Trèves se rapprocher très sensiblement à proximité de la levée de terre médiévale où un élément de la première a été reconnu jadis : nous sommes là certainement tout proches de l'agglomération principale. Symétriquement. à l'Ouest, l'infléchissement, aujourd'hui pratiquement certain, de la route de Cambrai-Tournai en direction du grand monument fournit une limite analogue. ('es deux points sont séparés d'environ 700 mètres. Dans le sens Nord-Sud, où les indices restent plus vagues, cette dimension ne semble même pas avoir été atteinte : elle pouvait se borner à 600 ou 650 mètres; ce qui nous donne une superficie totale d'environ 40 à 45 hectares. Nous retrouvons ainsi curieusement un des chiffres d'Amiens, celui du plan primitif. Mais i Bavay les choses en restèrent là ; Bagacum ne connaitra pas le mème développement que Samarobriva, ni même exactement celui de Gesoriacum-Bononia. Ces deux centres l'ont toujours emporté par leur rôle économique sur la capitale plus modeste des Nerviens. Lieu de passage obligé certes, mais sur des routes dont l'importance stratégique l'emportait. sur la fonction économique. Au surplus, le caractère urbain de la civilisation romaine n'a peut-être pas rencontré chez les Nerviens un terrain des plus favorables : les remarques faites par Jules lésar sur leur caractère farouche pourraient le laisser croire.

Dans son apparence extérieure, la ville du Haut-Empire ne devait pas différer fondamentalement de ses voisines; maisons et bâtiments publics étaient sans doute très semblables dans leur style comme dans leur aménagement ${ }^{17}$. Mais un problème doit nous retenir ici : la ville du Haut-Empire posséda-t-elle un plan à damier analogue à relui d'Amiens? Les données disponibles sur ce point restent encore assez floues. Nous connaissons aujourd'hui sùrement trois rues de la ville antique ; elles couraient sur les côtés nord, est et sud du grand ensemble monumental et se recoupaient donc à angle droit. Cielle des trois qui a pu ètre étudiée le plus commodément descendait un vallon

(14) Découverte au lieu-dit le Bisuir, ef. E. Dessarmss, Monuments épigr. provenanl de Bavai (.Mém. Soc. agr. sciences el arts, bouai, t. XI) pl. III ; H. Bníver.f, Gallia, 19.43, p. 171, fig. 3, et Annales Cercle archéol. Mons, 62, 1955 , fig. 3.

(15) H. Bı́veitet, Dallages de marbre, mosaiques el peinlures murales i Bavai, dans Lalomus, 1956, p. 568, pl. XI, fig. 2 .

(16) Dans les puits de fondations qui ont servi à la construction du nouveau College Notre-Dame de l'Assomption, cf. rapport dans Gallia, 1961, p. 281 .

(17) Sur les habitations de Bavay antique, cf. H. Btivilit, Rev. Nord, 1956, p. 273 (les caves à niches; ;.atomus, 1956, p. 567 (dallages, marbres et stucs); Les Eludes Classiques, 29, 1961, p. 166 (hypocaustes). 
naturel, auquel correspond aujourd'hui en gros la rue des Gommeries, et son tracé s'accompagnait sous terre du cours d'un puissant égout, un des grands collecteurs de la $v^{\text {ville }}{ }^{18}$. L'existence des deux autres rues sur les côtés nord et sud est impliquée par les rangées de boutiques qui flanquaient le grand monument sur ses façades extérieures. L'empierrement de l'une d'entre elles a d'ailleurs été relevé sous la tour d'angle nord-ouest de l'enceinte du Bas-Empire ; elle se prolongeait vers l'Ouest de façon à rejoindre la route Cambrai-Tournai, comme nous l'avons déjà dit.

Quelques autres indices viennent appuyer l'hypothèse du plan à damier. Les vestiges d'habitations, nombreux, on l'a vu, dans le périmètre de la ville actuelle, semblent pouvoir s'adapter pour l'ordinaire à un plan de ce genre. Tout récemment d'ailleurs, comme veut bien m'en informer II. le Chanoine Biévelet, un élément de rue a été constaté au Nord de la Grande Rue ; il peut prolonger en droite ligne celle dont nous venons de parler en dernier licu. Si cette donnée se confirmait, l'existence du damier serait pratiquement certaine.

L'idée de ce damier, quand on examine un plan de la ville, se trouve soutenue par la forme mème du grand ensemble monumental : on retrouverait là très volontiers la valeur d'une ou plutôt de deux insulae. Dans le sens Nord-Sud, nous obtiendrions une donnée à peu près certaine, la dimension de l'insula correspondant à l'espacement des deux rues qui longeaient les boutiques nord et sud; on peut fixer cette dimension a 65 pas romains, ou 95 m. 56. Dans le sens Ouest-Est, la situation est moins claire. D'un côté, le grand ensemble est flanqué à son extrémité ouest d'une "basilique" qui fait saillie sur le rectangle principal : cette anomalie peut avoir causé quelque trouble dans le tracé des voies Nord-Sud a cette place. D'un autre côté, il est certain depuis longtemps que le grand monument 'omportait deux moitiés dissemblables, lesquelles pouvaient être ou. peut-être, devaient être séparées par une rue transversale Nord-Sud ${ }^{19}$. La longueur exacte de chacune de ces deux parties n'est pas encore connue : on voit seulement qu'elle n'était pas identique de part et d'autre.

Le forum - et il ne peut guère faire de doutes qu'il s'agisse du forum avec ses deux moitiés, politique et marchande - occupait dans le plan tel que nous l'avons délimité une place assez centrale, encore que la moitié que nous sommes portés à considérer comme réservée à l'administration, la moitié ouest, se trouve placée en retrait par rapport au centre de l'agglomération; cependant, à Augusta Raurica, la situation du forum et en particulier celle de la Basilique est encore plus excentrique.

L'histoire de Bavay est jalonnée par quelques indices chronologiques. Le premier en date est fourni par la fameuse dédicace à Tibère qui, selon les ingénieuses déductions de J. Heurgon, doit

(18) On n'a pas republié récemment de plan d'ensemble mis à jour du champ de fouilles de Bavai ; cf. provisoire-

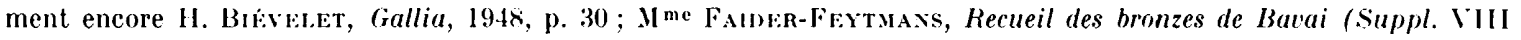
de (iallia) plan h. t., a complèter par plans partiels parus dans les rapports de Gallia. Pour l'égout, cf. HI. BIÉVELET, IIommages I.. Herrmann (Coll. Lalomus, 44) (1960), p. 152.

(19) L'integration du forum dans le plan d'une ville antique semble avoir souvent soulevé des difficultes en raison de l'extension de ce monument : d'ordinaire, il occupe la valeur de plusieurs insulae; parfois il est partagé par une rue transversale, cf. les plans comparés présentés par P. M. Deval, Paris anlique, pp. 134 et 135. A Callev'rSilchester, le for'um est situé au centre d’un groupe d'insulae sans être directement contigu à aucune des rues du quadrillage, cf. C. Boox, Roman silchester. A Baray, la situation reste énigmatique ; les dégagements assez importants pratiques au Nord du monument, sur la rue de Valenciennes, nont pas fait apparaitre de rue Nord-Sud. 
se placer en 4 de notre ère ${ }^{20}$ : c'est la date que nous retrouverons et pour le mème personnage ì Boulogne. Bagacum gallo-romain existait donc dès le début de l'Empire; sa fondation doit, en réalité. ìtre liée à l'élablissement du réseau routier dans lequel il occupe une place si éminente et cet établissement doit remonter à une dale ancienne - nous y reviendrons encore. Mais ce n'est là qu'une indication très générale pour l'histoire du développement urbain de la capitale des Cerviens.

La ville existait-elle du temps de l'indépendance? En tant que ville ou oppidum. on peut le nier sans trop de hardiesse. César ne mentionne pas un seul centre urbain ou fortifié pour cette peuplade farouche et imperméable aux influences méditerranéennes. La seule place forte des Nerviens qui nous soit connue est celle du camp d'Avesnelles aujourd'hui si lamentablement détruit ${ }^{21}$ : c'était sans doute un refuge plutòt qu'une capitale et qui pourait représenter un ultime essai de résistance désespérée. Cependant le nom même de Bagacum prouve le caractère indigène de l'endroit ; mais il n'y eut là sans doute qu'un village ou un hameau dont l'élévation au rang de chef-lieu d'une civitas, en dépit d'une situation lout à fait excentrique dans le territoire des Nerviens, est une manifestation de la volonté toute puissante du maitre romain ${ }^{22}$. Rien ne nous renseigne plus: aujourd'hui sur l'emplacement exact de ce village indigène ; on peut douter que la ville romaine lui ait été simplement superposée. L'emplacement définitif au sommet d'une pente qui descend doucement vers le ruisseau de Bavay, en une situation commode et salubre à la fois, révèle assez l'inspiration romaine du choix.

Le double forum, au témoignage de certaines découvertes de céramique, appartient au milieu du II $^{\mathrm{e}}$ siècle ; de façon générale d'ailleurs. la céramique recueillie à l'intérieur de la ville actuelle est gallo-romaine et plutòt tardive. Cependant c'est dans le for'um mème que fut découverte la dédicace a Tibère et certains aménagements du sous-sol de la "basilique " semblent permettre de reconnaître deux états pour cet édifice. dont l'un pourrait remonter au $\mathrm{I}^{\mathrm{er}}$ siècle ${ }^{23}$. De la céramique arrétine a élé découverte tout récemment, enfin. en quantité assez abondante, sur la limite sud de la ville, en bordure de la route de Saint-Ouentin ${ }^{24}$ : le site commençait donc à se peupler de facon sérieuse dès le début de notre ère. Le forum monumental apparait comme un embellissement postérieur et il n'est finalement pas exclu qu'il fasse partie d'une réorganisation générale du plan de la ville - à un moment qui peut correspondre à l'agrandissement constaté à Amiens.

Cette ville fut viclime des invasions du milieu du nue siècle, certainement dès le premier assaut déja ${ }^{25}$. I'n document assez pathétique de l'époque est la petite tombe à incinération découverte au sud-kst de la Grand'Place, rue des Recollets : un antoninianus de Claude le Gothique de 269 fournit un terminus post quem ${ }^{26}$. La ville est en ruines à cette date, comme ses voisines. Un rempart s'élevait-il déjà tout auprès? La fortification rapide de celte plaque tournante apparaît, en tout cas, comme des plus probables. La déchéance de la ville fut d'ailleurs profonde : de ses 40 hectares primitifs elle se trouve amenuisée à une surface qui atteint tout juste 4 hectares -.. c'est le chiffre le plus faible connu dans la région ${ }^{27}$. On peut croire qu'elle fit office dés lors de place forte plus que de centre

20) Cf. J. Hecroox, Antiquile Classique, 1948, p. 323.

(21) Cf. Ch. Cron, L'Avesnois préhist. gaulois, gallo-romain el franc, p. 39. Ce camp comportail, en effet, un murus gallicus et si les deductions de Wheeler-Richardson dans leurs Hillforts of Norlhern france sont correctes, ce (amp pourrait devoir son existence à la Conquête même.

(29) On peut comparer la situation tout aussi excentrique de Cassel dans le territoire des Minapiens ; là, ce sont des raisons strategiques qui ont du dicter le choix.

(23) Pour la dédicace à Tibère, cf. ci-dessus, p. 87 el n. 20. Pour les remaniements possibles de la Basilique, Gallia, 1948, p. 309.

(24) C. le rapport de fouilles de Gallia 1962. On a trouve dans ces fouilles des restes de puissants madriers de chène qui appartenaient à une installation énigmatique - qui pourrait dater des débuts de la ville romaine. Le tout se trouvait malheureusement entre $3 \mathrm{~m}$. 50 et 5 mitres sous terre.

(25) Cf. J. Hutcion, Gallia, 1954, p. 142, sur les trésors qui datent ces événements : à noter celui trouvé à l'interrieur du tracé de l'enceinte avec des pièces de Maximien el de Dioclétien.

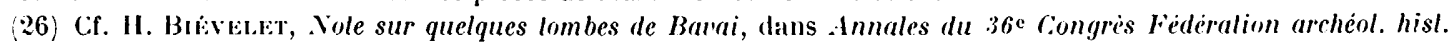
de Belgique, 1956, p. 149 et fig. 1.

27) Il se peut que Cassel se soit trouvé réduit de mème : ces faits expliqueraient pourquoi ces deux centres du 
urbain. Bayacum. qui n'est plus connu des textes antiques les plus lardifs, a pu disparaitre dés le début du ve siècle - là encore, la date de 406-407 apparait comme un terme possible. L'avance des Francs sur Cambrai marqua en toul cas le point final.

\section{BoLlogNe-BONONAA-GESORIACLM.}

Le site de Boulogne n'a jamais été l'objet de fouilles méthodiques; il n'a mème pas été possible de profiter, comme à Amiens, des destructions de la dernière guerre pour préciser nos connaissances très fragmentaires. Toutefois une foule de découvertes fortuites, assez soigneusement relevées au cours $\mathrm{du} \mathrm{xIx}^{\mathrm{e}}$ et au début $\mathrm{du} \mathrm{xx}^{\mathrm{e}}$ siècle, permettent de restituer le visage de la ville antique, au moins dans ses grandes lignes ${ }^{28}$.

L'histoire du site est complexe. mais quelques données littéraires et épigraphiques. que nous a values l'importance de l'endroit. nous fournissent d'abord un cadre chronologique assez précis. Boulogne gallo-romain apparaît pour la première fois dans une lettre de Tibère au sénat et au peuple de la ville phrygienne d'.lizanoi ${ }^{29}$; nous apprenons que le fulur empereur séjourna en 4 de nolre ire, au cours d'un voyage vers la Germanie. dans la ville de Bononia. Si. par ailleurs. les déductions de J. Heuronon au sujet d'un texle de Florus sur les pontes Drusi sont justifiées. comme on le croira volontiers, le lerminus post quem dont nous disposons se trouverait reporté aux années 12 a 9 avant J.-C. ${ }^{30}$. Dans ce texte. la ville est déjà citée sous le nom de Gesoriacum qui lui restera pendant près de trois siècles. Ce premier changement de nom peut correspondre à une organisation nouvelle qu'on peut mettre en relation avec la construction du fameux phare sous Caligula ou alors avec la conquête de la Bretag̣ne par Claude. L'installation de la Classis Britannica à Gesoriacum a dû faire préraloir rette derniere appellation.

Touveau changement de nom vers 300 : Gesoriacum disparait au profit de Bononia. L'épisode de la révolte de Carausius, terminé par la reddition de la ville en 293 , se trouve ainsi sanctionné en quelque sorte par le vainqueur, Constance Chlore; mais on dira sans doute aussi bien que le changement traduisait une modification brutale des données militaires: c'est la ville haute, Bononia, resserrée dans sa ceinture fortifiée, qui redevient le centre à la place des quartiers de la ville basse réunis sous le nom de Gesoriacum, quartiers dont une partie fut d'ailleurs sacrifiée ${ }^{31}$.

La fin de Bononia oceanensis, comme l'appellent les monnaies, se place au début du ve siècle. La ville est mentionnée la dernière fois comme base de Constantin III, qui y débarqua avec les troupes ramenées de Bretagne ${ }^{32}$. L'entreprise de l'usurpateur, qui fut fatale à la province abandonnée, le fut sans doute tout autant à Boulogne. Bononia est inconnue à la Nolitia dignilatum, tout comme la Classis Britannica. Le nom mème de Gesoriacum est évincé à jamais et à son emplacement s'élèvera

Haut-Empire céderent la place, au Bas-Empire, à Tournai et à Cambrai. L'ne autre ville a peut-ètre ou sans doute disparu comme centre urbain à cette date : Augusta Viromanduorum; il n'est pas exclu que l'ancien oppidum de Vermand ait repris alors son ròle de centre de la civilas. Ce n'est que beacoup plus tard que Saint-Quentin reprendra la tradition d'A ugusta.

(28) C.. notre mise au point parue dans Rer. Nord, 1960, pp. 363-379.

(29) L.es textes concernant Boulogne antique ont été soumis à un nouvel et pénétrant examen par J. HELRGiox: I.es problemes de Boulogne, Rev. éludes anciennes, 50, 1948, p. 101 ; Encore un probleme de Boulngne: le pont de Irusus, ibid., 51, 1949, p. 324; De Gesoriacum it Bononia, Iommages Bidez-Cumont (Coll. Latomus, II, 1949), p. 127. Pour la lettre de Tibere, cf. le premier article cité.

(30) Reu. éludes anciennes, 51, 1949, p. 32.4; cf. Flont's, II, 30 (IT; 12, 26).

(31) C.f. notre étude Rev. Nord, 1960, pp. 377-379, sur la date du rempart et les conditions dans lesquelles il fut ilevé.

(32) C. notre étude citie pp. 364-370. Sur le texte de saint Jéròme et son interprètation, cf. Chr. Colrois, I.es Vandales, p. $1 \cdot 2-51$; la chronologie de ces événements reste malheureusement flottante, mais Courtois a ell raison de souligner la valeur du texte citi. 
un hameau de Saxons ou de Francs, révélé par le nom germanique de Brequerecque ${ }^{33}$. Le port ne revivra que sous Charlemagne. dans la lutte contre le péril normand.

Cette histoire est donc marquée par l'alternance de deux noms, (iesoriacum et Bononia. alternance qui recouvre un déplacement du centre de gravité : de la ville haute, on passe a ville basse et vice-versa. Sur ce point, tout le monde est pratiquement d'accord aujourd'hui. Mais si l'identification de la ville haute avec Bononia ne pose pas de problèmes majeurs, la localisation et la délimitation de Gesoriacum ont paru plus difficiles. J'ai discuté ailleurs. plus en délails, de cette question et une présentation plus brève des données et des résultats suffira ici ${ }^{34}$.

Si l'on cherche à définir l'extension de la ville du Haut-Empire (fig. 3), on ne dispose que d'un indice d'une précision relative : cette extension se trouve révélée, comme il arrive ailleurs, en négatif en quelque sorte, par celle des nécropoles et pour ces dernières nous possédons des indications suffisantes pour notre propos. Ene première zone de cimetières courrait, au Nord-Ouest, les pentes entre le vallon des Tintelleries et l'enceinte de la ville haute ; elle s'étendait sans doute sur tout le còté nord-est de cette dernière; quelques monuments bien identifiés attestent l'existence de cette nécropole sous le Haut-Empire. A la même période appartient le cimetière antique fouillé en $18939-95$ par le Dr E. T. Hamy à l'emplacement de l'actuel cimetière de l'Est, en un groupement dense, au Nord de la rue du Vieil-Atre au nom révélateur. Troizième zone enfin, celle qui bordait de part et d'autre la route de Paris aux portes du faubourg de Brequerecque jusqu'à hauteur de l'ancien abattoir. Ces trois nécropoles permettent de tracer pour la zone résidentielle un rectangle allant du rempart nord-ouest de la ville haute jusqu'au val Saint-Martin au Sud-Est, de la Liane au Sud-Ouest au chemin d'Echinghen et au front nord-est de la ville haute. C'est une superficie qu'on peut évaluer grossièrement à 40-j) hectares et qui courrait un terrain accidenté et raviné. Que cette zone fût résidentielle est prouvé par les vestiges signalés aussi bien dans la ville haute que dans le quartier de Brequerecque. Mais il convient surtout d'ajouter une remarque importante : la mème zone abritait les installations de la Classis Britannica. Des restes certains de constructions ot un nombre considérable de briques et tuiles au sigle de la Classis ont été découverts sur une assez vaste surface au pied de l'angle sud de l'enceinte médiévale. Des docum'nts analogues ont été signalés aussi à l'autre extrémité de la même zone, "au val du val Saint-Martin ". Ainsi se trouve délimité ce qu'était Gesoriacum: tout le quartier construit et habité en dehors de l'actuelle ville haute.

Mais ainsi se trouve Iranché aussi un problème discuté pendant longtemps : celui de l'emplacement du port antique, du moins du port principal, qui doit èl re cherché dans ce qu'lirnest Desjardins ${ }^{35}$ appelait. avec une juste appréciation de la situation. "l'anse de Brequerecque ". Que. contrairement. i l'avis de certains, le port ne fût pas installé à la sortie du vallon des Tintelleries. comme ce fut le ras au Moyen Age. est prouvé par toutes sortes d'indices. Ine nécropole. nous l'avons vu. s'étendail

(33) Autour de Brequerecque (mot dans lequel il faut sans doute reconnaitre le vocable grermanique signifiant inlise ; cf. Dunkerque), les toponymes germaniques sont fréquents, comme tout autour de Boulogne, fait noté depuis longtemps ; cf. Pitendal, Ostrohove, Bertinghen, Echinghen, etc.

3.1) C.f. etude cité, pp. 372 et suiv.

(35) E. DEs.mandss, Géngraphie hisl. el adminislr. de la Gaule rom., t. I, p. 38.3, avec un plan aux restitutions trop precises et contestables, reproduit par Grixike, Wannel, II, 2, p. 527, fig. 169. 


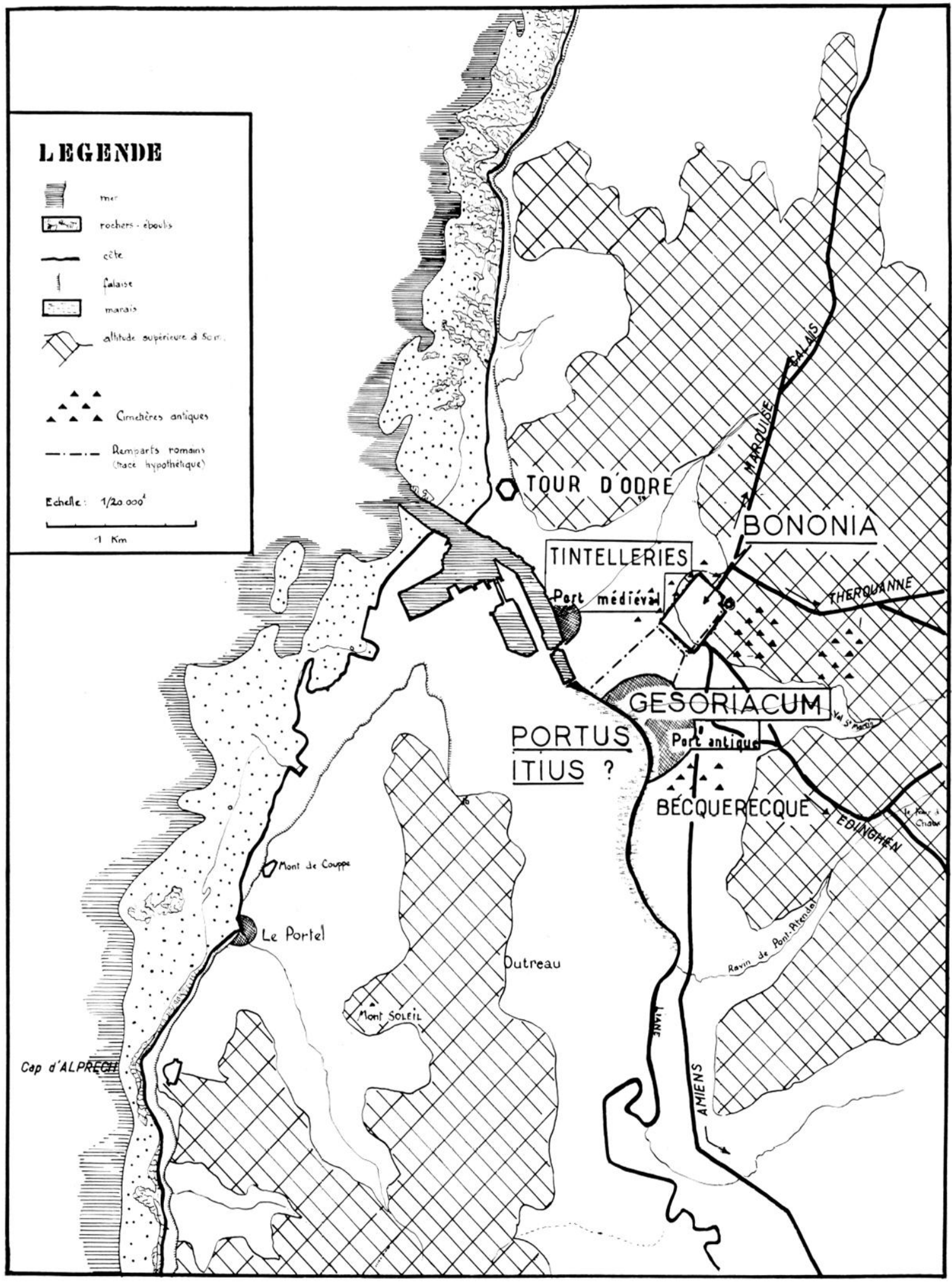

Fig. 3. - Le site de Gesoriacum-Bononia-Boulogne.

(La zone des ports à titre indicatif). 
sur le flanc méridional dudit vallon du còté de la ville haute. Au Bas-Limpire. la ville basse, elle aussi entourée de murailles, ne rejoint nullement le vallon en question - ce que fera, au cont raire, et par un déplacement caracléristique. le quarlier correspondant de la ville médiévale ${ }^{36}$. L'anse de Brequerecque, qui s'était ensablée au cours des siecles comme le reste de l'estuaire de la Liane, était devenue inutilisable au Moyen $I$ gee ${ }^{37}$, mais son Iracé apparail encore el de façon frappante sur les cartes qui furent dessinées de la ville au $\mathrm{XvI}^{\mathrm{e}}$ et au Xvif ${ }^{\mathrm{e}}$ siècles (fig. 4). Il a fallu. pour que ces faits fussent méconnus. l'obstination trés caractéristique de ceux qui ont de la peine à reconnaître un phénomène classique dans la vie des siles urbains : le déplacemenl des cent res. L'exemple d'Amiens. tel que nous l'avons reconst il ué. en est pourtant aussi une belle illust ration. C'est une aut re discussion. tout aussi vaine, qui a fait chercher le Portus Ilius de Jules César en toutes sortes d'endroits, saul sur l'estuaire de la Liane - qui présentait en ce temps la des possibilités disparues depuis ! On placerait volontiers ce port sur la rive gauche de la rivière. du còté où le gxéographe Plolémée signale un Ition aliron, nom qui étail peut-ìtre celui de tout l'éperon culminant aujourd'hui au Iont soleil el sur lequel Jules Céasr a pu installer son quarlier qénéral ${ }^{33}$.

Ln aveuglement analogue a sans doute fait méconnaître le caractère véritable de la ville haute du temps du Ilaut-Empire : on y reconnaissait une curieuse mixture de quartier résidenticl et de castrum militaire. De fait, le caractère même de quartier résidentiel est suffisamment prouvé par les vestiges découverts au cours du siècle dernier sous l'église Notre-Dame et sur les deux còtés de la rue de Lille : "temple ", "balneum " et "édifice religieux ». La liaison de ce quartier avec Gesoriacum était de même en quelque sorte illustrée par les restes de murs qui. dans une cave de la rue Saint-llartin, passaient encore sous le rempart médiéval. Les habitations pouvaient très bien se poursuivre du côté sud-est le long du Chemin d'Echinghen (rue Boucher-de-Perthes et suivantes), jusqu'à Brequerecque ${ }^{39}$. On peut même penser que les demeures riches recherchaient plutôt les hauteurs que les fonds en bordure de la Liane, et cela de la ville haute jusqu'au Val Saint-Martin.

I'ne autre donnée, dont on fait traditionnellement état pour la ville haute. est celle du quadrillage romain qui apparaitrait encore clairement dans le tracé des rues actuelles ${ }^{40}$. Les rues, en effel, qui joignent aujourd'hui, de façon plus ou moins rectiligne, les quatre portes de l'enceinte médiévale représenteraient le cardo et le decumanus du damier antique (fig. 5). Cette façon de voir néglige fâcheusement deux données importantes. La première concerne le principe même de l'établissement d'un cardo et d'un decumanus. Cette croix de voies principales, avec leurs parallèles d'importance secondaire, a trìs évidemment présidé au plan d'un certain nombre de villes du HautEmpire, ainsi à Amiens el sans doute à Bavay, on l'a vu. Mais il paraîl à peu prís élabli aujourd'hui que le système fut abandonné au Bas-Empire. où les villes ont. tendance à s'organiser autour d'un

(36) Sur le developpement médiéval, cf. P. Ilísor, Essai sur le dételoppement urbain de Boulogne, Bullelin Sior. acad. Bouloyne, XII, 1929-33, pp. 284 et 294 , et Rev. Nord, 1932, p. $\times 1$. l.a ville hasse médiévale ne conserva dans ses murs yu'une fraction de la ville basse antique et prit une extension assez considerable du eòte des Tintelleries.

(37) Sur le port médiéval et des siécles suivants, cf. P. Hit.10T, Rer. Nord, 1958, p. 27 et fig. - - Ine étude sur l'histoire du port a paru dans te recueil Bunlugne el le Boulonnais, 1899, p. 695, due a J. Voisin ; une série de vues anciennes sont publićes la-mème, fị. 5,6 et 10 ; on distingue toujours la fameuse anse et les "prairies inondées " qui devaient fournir un plan d'eau suffisant. Yous reproduisons ici une vue du siegge de la ville par Henri VIII d'Angleterre, vue assez éloquente elle aussi, cf. fig. 4.

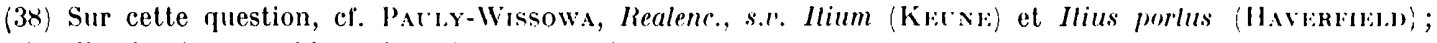
pour la localisation topographique, P'Tolínili, II, 9, 1.

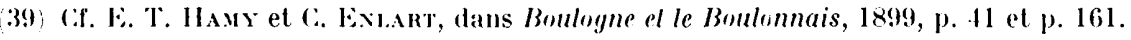

(40) ef. p. ex. pour la doctrine traditionnelle, E. T. HAMy à l'endroit cite, ou P. Hiblot, Origines de la llaule l'ille de Bomlogne, dans Eludes historiques à la memoire de Roger Rodière (19.7) (Mem. Pas-de-Lalais, \, 2), p. 26 et suiv. 


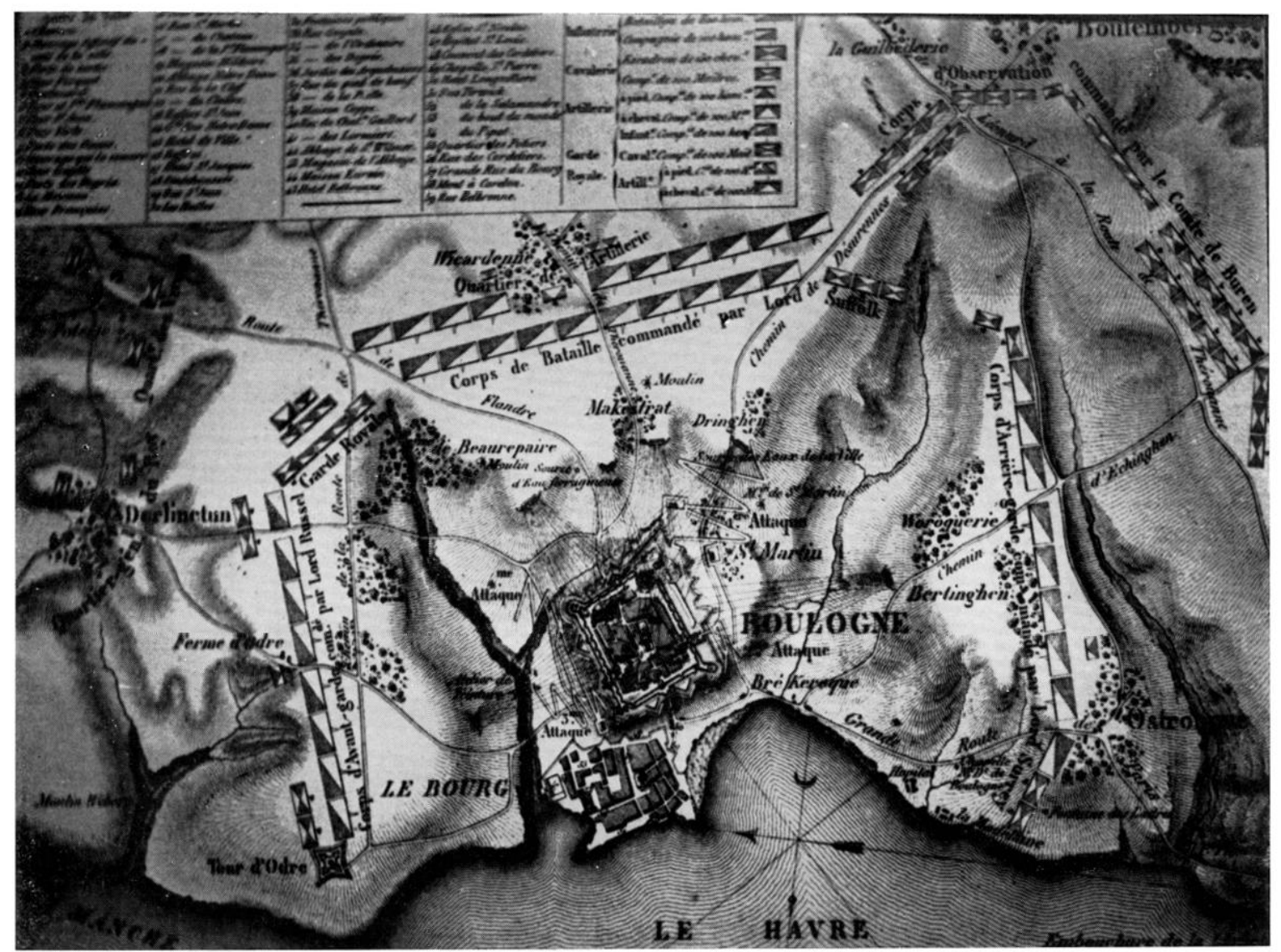

Fig. t. - Plan de Boulogne et environs en 15.14.

axe principal. Gue dirons-nous donc pour la ville haute moderne de Boulogne. qui se ratlache lris: directement. dans sa délimilation, à celle du Bas-Empire précisément 41 ? Comment peut-elle conserver l’image d'un cardo el d'un decumamus (fu'elle n'a connus ni au Haut-Empire ni au Bas-Empire?

L'arbil raire des vues lraditionnelles n'est pas moins flagrant quand nous abordons la seconde donnée : le probleme des portes. Pour l'une d'entre elles, celle des Iunes, on peul croire qu'elle n'a pas existé dans l'Intiquilé où elle élail pratiquement sans utilité La ville haute du Bas-Empire possédait nécessairement deux portes : l'une menant vers la ville basse. l'aulre menant vers le dehors. sur la route de Calais el sur celle de Paris. Pour celle-ci. un emplacemenl proche de celui de la Porte (iayole reste probable. proche puisqu'il semble que celle porte. aussi bien que la muraille adjacente. ne remonte pas plus haut que Philippe Ilurepel. El la mème date convient au rempart sud-ouest actuel avec la Porte des Inegrés : rien ne prouve que la communication entre ville haute el ville basise se soit trourée dans l'Intiquité. non pas à l'emplacement. mais dans l'axe simplement de cetle Porte. Quant a la quatrieme ouverture. celle de la Porte Neuve fou de Calaisi. son nom meme trahit une oriqine non anlique.

Il vaut ainsi mieux renoncer au cardo et au decumanus chers a la tradition locale. cependant. un examen du plan de la ville haute actuelle fait apparaitre quelques 
particularités qui méritent de retenir l'attention (fig. 5). Voici d'abord l'orientation de l'église Notre-Dame, si curieusement en oblique par rapport à la muraille nord-est de l'enceinte - qui, elle, englobe de ce côté un noyau antique. Sans doute cette orientation pourrait-elle trouver son explication dans les phases diverses, et peut-être assez récentes, que connut cet étrange édifice. Mais la vérité est que la mème orientation se retrouve apparemment de l'autre côté de la rue de Lille, dans le départ de la rue de l'Oratoire et dans celui de la rue Saint-Martin (dont les cours se trouvent infléchis ensuite vers la Place ou vers la Porte Gayole). Dans la mème région aussi, une orientation perpendiculaire est donnée par les rues du Château et de la Balance, comme naturellement aussi par le Parvis Notre-Dame, et aussi, semble-t-il (le fait serait des plus remarquables), par la rue d'Aumont qui mène à la Porte Gayole. Ce dessin orthogonal et divergeant avec obstination de l'orientation de l'enceinte surprend éridemment; il peut trouver son explication dans des survivances plus anciennes, qui remonteraient au Ilaut-Empire. Ce qui a pu assurer sa conservation locale, c'est la persistance d'un ou de deux monuments importants, qui n'ont pu être ruinés de fond en comble par les barbares du ire siècle. Le fait mérite pour le moins d'ètre signalè et, en attendant d'éventuelles découvertes favorisées par la chance, on peut le noter avec les réserves nécessaires sur le bien-fondé desquelles nous avons nous-même attiré l'attention ci-dessus à propos d'Amiens.

Si ces observations venaient à ètre confirmées, nous aurions encore la preuve de l'existence à Boulog̣ne mème d'un plan à damier. Cé plan aurait dépassé naturellement les limites étroites de l'enceinte actuelle, en direction du Sud-Est notamment; le chemin d'Echinghen a quelques chances de répondre à la même orientation générale. Pour le reste cependant, il n'est nullement assuré qu'un damier uniforme ait englobé toute la future ville basse ou encore le quartier de Brequerecque situé autour du port : des nécessités pratiques ont pu ou même dù entraîner dans ces secteurs des orientations particulières ${ }^{42}$. C'est précisément le rôle qu'a pu jouer le port dans l'élaboration du plan urbain qui nous échappe aujourd'hui le plus complètement. Ce qui reste cerlain seulement, c'est que ce port a donné à l'agglomération un développement analogue à ceux que nous avons rencontrés précédemment pour les deux chefs-lieux de civilas que furent Amiens et Bavay ${ }^{43}$.

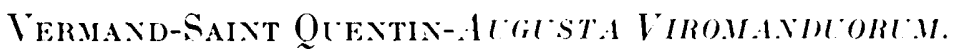

Nous avons rencontré jusqu'à présent deux cas dans le passage de la Ciaule indépendante à la province romaine, celui de la permanenee du site avec Amiens et Boulogne, celui de la création nouvelle avec Bavay. I'n autre cas bien connu et, en effet, très fréquent est celui de l'abandon d'un oppidum gaulois au profit d'un site proche, mais plus conforme aux conceptions romaines, plus adapté à une vie pacifique et civilisée. Nans la France du Nord, les exemples ne manquent pas : Beauvais, Arras, Soissons et Reims. La fondation nouvelle est sanctionnée parfois d'un nom nouveau évocateur de la volonté du maître de

(12) Autour de l'anse de Brequerecque, la voie antique correspondant à l'actuelle route de Paris a éte constatie plusieurs fois : son tracé semble avoir íté à peu près parallèle, mais un peu en contrehas vers la liane.

(13) La ville suit de même l'évolution de ses voisines au Bas-Lmpire; si Amiens se contente d'une superficie denv. 10 hectares, la ville haute de Bonlogne en comptera 12-13, auxquels il conviendra dajouter quelenes hectares pour la ville basse. 


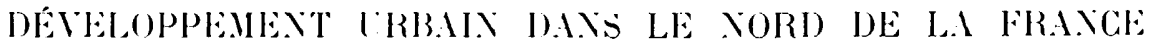

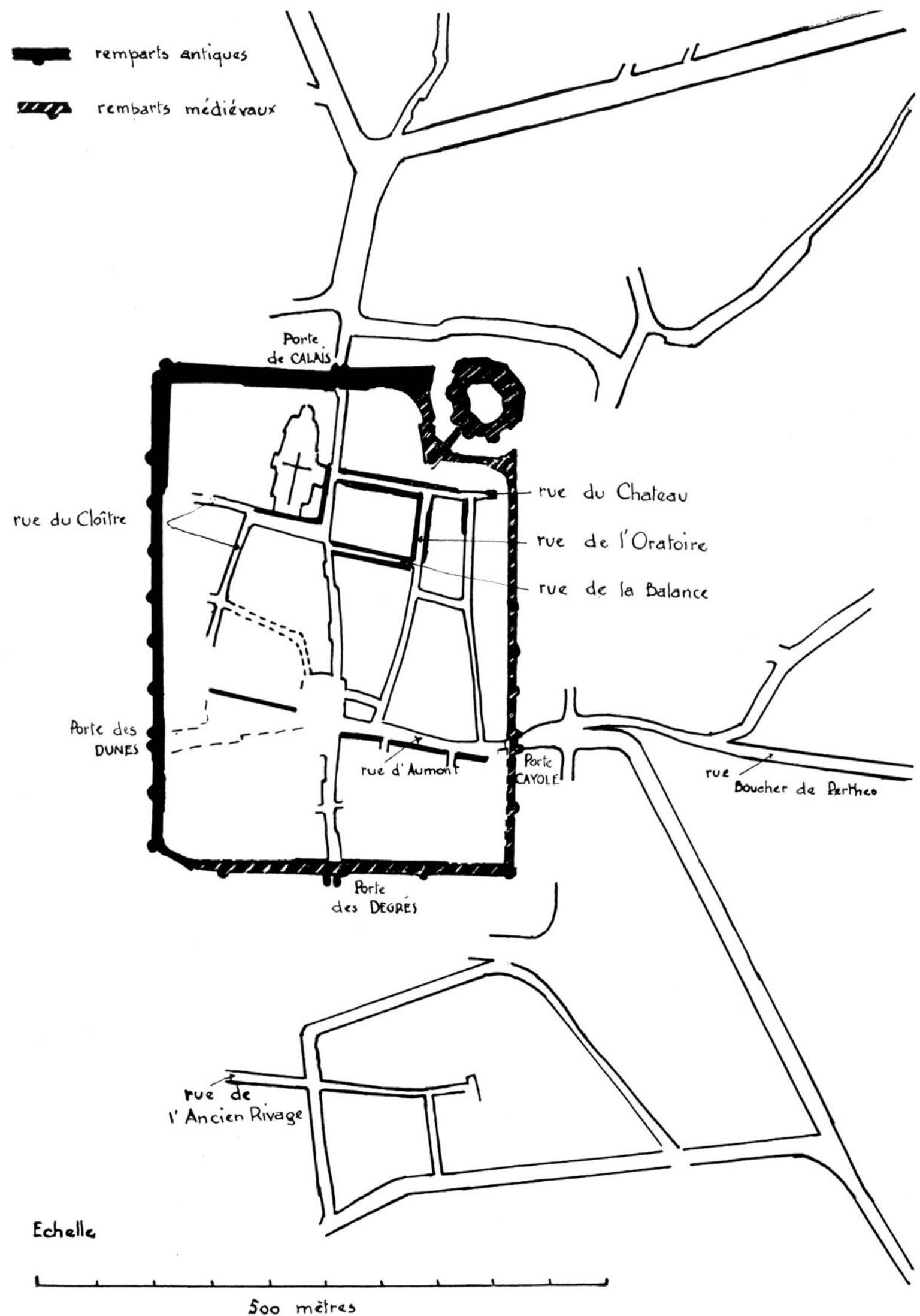

l.ig. 5. -. La ville haute de Boulogne : traces possibles de voies romaines. 
l'empire : à Braluspantium sucrède Caesaromagus, à Noriodunum. Augusta Suessionum et à Vermand, Augusta Viromanduerum. Mais ce dernier eas est plus romplexe et comme il a l'avantage de révéler des hésilations de la part des organisaleur's romains de la ciaule. ii vaut la peine de s'y arreter.

C'étail. au siecle dernier. parmi les savants locaux, une discussion traditionnelle aussi

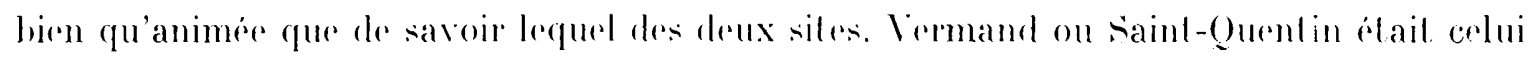

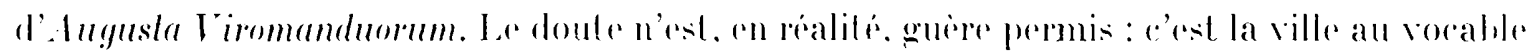

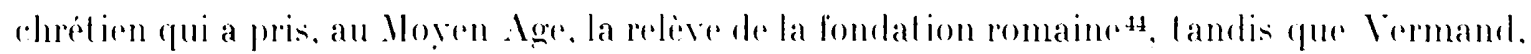

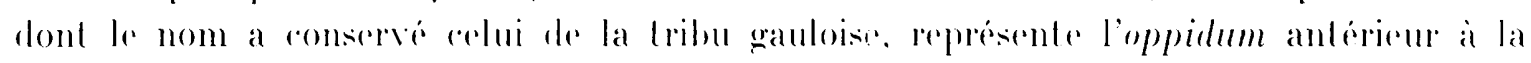
ronquete : la puissante levé de lere qui contoure loujours la localite moderne remonte au moins partiellement a cente haute antiquiti.

Jusque la rien que de hanal sans doute, mais il suftil de regarder une carte el de reperere Je trace des voles romaines pour constater une anomalie assez singuliere fig. 6j. Tout se passe. en effet, comme si Vermand servait de point de depart a certaines routes. SaintQuentin a d'autres. Ainsi. dans le sens . Cord-sulul la priorite revient a cette derniere pour

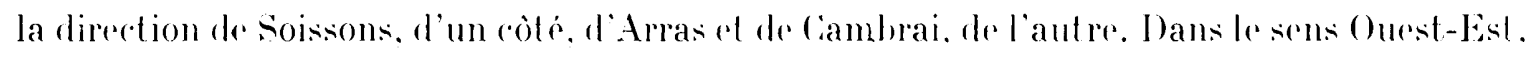
cest Vermand qui tient la tète : e'est lai que viennent se rencontrer deux des plus beelles

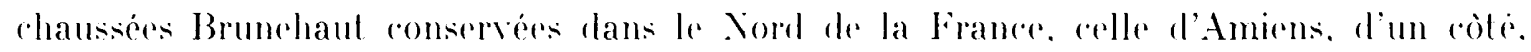
celle de Bavay, de l'autre; en si. naturellement. on venant dimiens. on pourait continuer en ligne direste vers Saint-()uentin. il n'on allail pats de meme quant on venait de Bavay, où un demi-tour à gaurele s'imposail a une dizaine de kilometres de la ville moderne.

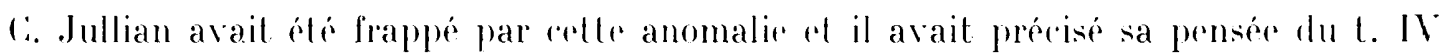
au 1. VI de sa monumentale Ilisleire de le Cianle. Après avoir parlé d'u une rectifieation locale pour adapter un ancien chemin à une rapitale nouvellen, il caresse pour finir l'idée d'une grande artere (Ouest-Est, Paris-senlis-Bavay, dont le tracé premier pourrait remonter a l'époque de l'Indépendance et soulignait en tout cas la place de Paris-Lutèe dans le Nord de la Ciauld ${ }^{45}$. Le grand historien sacrifiait ainsi a deux de ses idlées favorites : la permanence des chemins gaulois que les Romains se seraient le plus sourent contentés d'aménagerer en voies empierrées, le ròle ancien de Paris. Xi l'une ni l'autre de ces idées ne sont suffisamment étayées par les faits et nous pouvons en faire abstraction. si nous cherchons à comprendre le raison d'ètre du binôme Vermand-Saint-Quentin. le's deux sites apparaissent comme rivaux dans le systime routier romain : il s'agit de determiner les phases chronologiques de. cette rivalité.

Commençons par Vermand, qui domine un axe ()uest-List. Ylais il ne s'agit pas, comme le pensait c. .Jullian, d'un axe dont le hut était de redier Paris an Rhin par Senlis et Bavay. lin tel ròle n'aurait pu échoir à Lutèe qu'à un seul moment de son histoiré, à l'époque vù, pour la premiere fois, clle derient une capilate, du moins pour le Nord de la ciaule : less

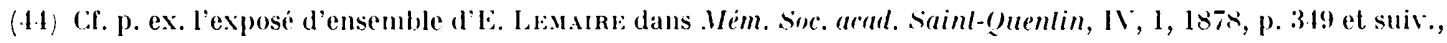
avec une carte de J. Pilloy, p. 39.4, dapres laruelle nous avons établi notre fig. 6. Fait caractéristique : un des quartiers de Saint-Quentin médiéval portait le nom d'Atousle (ct. l.l., p. 415).

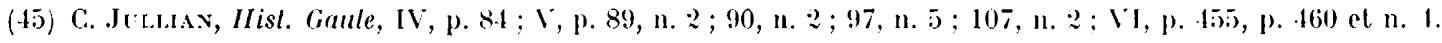
l.e dernier auteur qui s'est occupé du systime routier de la région parisienne ne s'est pas rallie aux thises de Jullian, cf. P.-... Duvar, Paris antique (1961), p. 2.14. 


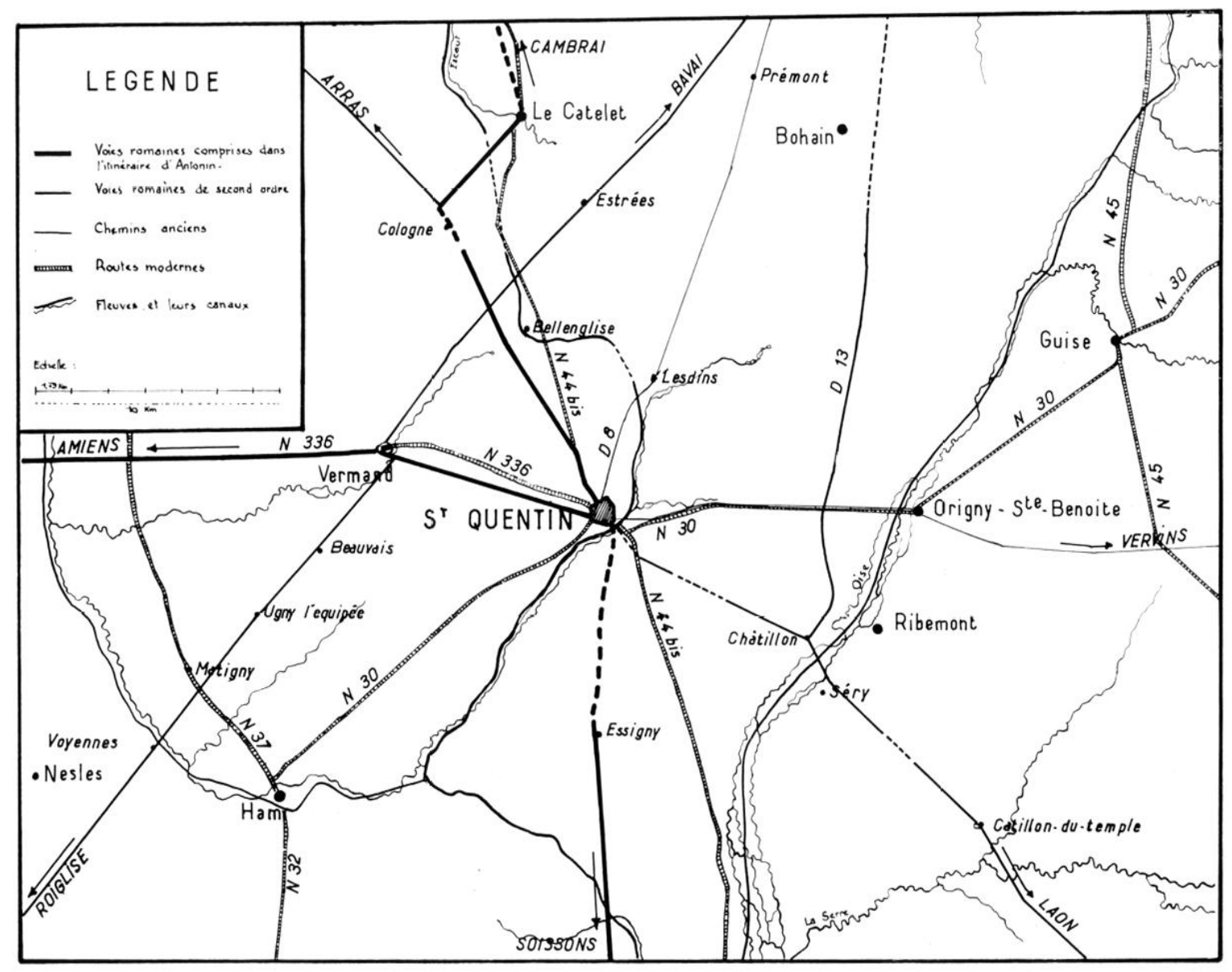

Fig. 6. - Le réseau routier romain dans la rigion de Saint-Quentin-Vermand.

années où Julien y résida. L'établissement de communications directes avec le Rhin eùt été à cette date tout à fait normal. Cependant, si l'on cherche les connexions de Vermand vers l'Ouest, on décourre autre chose. A proximité immédiate. en effet, un tronçon rectilignne de voie romaine a été reconnu ou proposé anciennement comme tel entre les localités de Beaurais, Lgny. Matigny et Voyennes au-delà de la Somme. On s'oriente de la sorte en droite ligne sur Roiglise (immédiatement au Sud-Est de Roye), dans lequel on a reconnu le Rodium de la Table de Peutinger ${ }^{46}$. Ce n'est pas tout : au-delà de Roiglise et en direction de Saint-Just-en Chaussée, quelques tronçons induhitables de voies romaines sont toujours apparents, de part et d'autre de Beurraignes, et, plus loin, de Boulogne-la-Grasse par Courcelle-Épayelle à Montgerain. Cette voie si obstinément dirigée vers le Sud-(Ouest devait rejoindre Beauvais, la ville la plus importante de la région, d'où les communications étaient de nouveau possibles ou vers Rouen ou vers (hartres.

(iet axe Ouest-Est, s'il est encore visible sur le terrain, ne figure pas dans la tradition antique. C'est le contraire qui est le cas pour le réseau de Saint-Quentin : l'axe nord-sud vers Cambrai-Arras, ou encore par Vermand à Amiens, est bien attesté ; la direction est 
toujours la même : le terme de la route, c'est Boulogne. Tout se passe comme si dans la solution qui correspond de fait aux premiers siècles de l'Empire, Augusta Virrmandunrum avait comme ròle essentiel - un rôle secondaire d'ailleurs dans l'ensemble du système routier - d'assurer la liaison du Sud vers le port de la Bretagne. L'axe Ouest-Est à cette date s'était révèlé, lui, comme périmé. Nous disons : "s'était rérélé "; il scmble préférable, en effet, de considérer cet axe ccmme une solution première, qui put ètre abandonnée par la suite. Ce sont des considérations militaires, peut-être, qui déterminèrent dans une première phase l'établissement de l'axe Ouest-Est. semblant avoir tenu compte du poids qui revenait au moment de la conquête, dans la Gaule indépendante, au peuple des larnutes et à celui des Belloraques. Nous serions prêts, pour notre part, à rapprocher un texte bien connu de Strabon $(I V, 6,11)$ qui fait passer la route de l'Océan établie par Agrippa expressément chez les Ambiens et les Bellovaques ; cette seconde mention, qui a toujours surpris, pourrait être le souvenir d'un systime dans lequel Beaurais occupait une place perdue par la suite. Pour ce qui est de Vermand en tout cas, quand la primauté de la route de Boulogne se füt imposée, elle entraina la fondation de la ville sur le passage de la Somme, Augusta, au détriment de l'ancien oppidum.

Est-il possible d'exprimer ces constatations en données chronologiques? L'organisation de la Gaule romaine se place, selon les vues traditionnelles. entre 27 et 12 avant notre ère. Ce sont là des dates facilement conciliables avec ce que nous savons d'Amiens, de Bavay el de Boulogne: la date la plus ancienne qui nous soit fournie par la tradition est, on l'a vu, celle des années 12-9 av. J.-C. pour les pontes Drusi. Mais nous avons vu aussi que l'emplacement donné aux différentes villes de la région était étroitement lié au système routier et ce dernier, selon la tradition. est une création d'Agrippa. Or $\Lambda$ grippa a été deux fois en Gaule, une fois entre 39 et 37 et une seconde fois en 19 avant nolre ère. On est tenté de placer au moment du premier voyage le système dans lequel Vermand occupe une place éminente et au second la solution définitive avec la fondation d'Augusia Viromanduorum ${ }^{47}$. Ces deux dates ne semblent pas devoir être rejelées sous prétexte qu'elles sont trop " hautes ". D'une part, en effet, des raisons militaires favorisaient naturellement l'établissement rapide d'un réseau roulier qui a pu précéder le statut des civilates. D'autre part, au choix de l'emplacement pour une agglomération urbaine n'a pu succéder qu'avec une cerlaine lenteur l'exécution matérielle.

Les données que nous avons réunies ci-dessus permettent-elles quelques conclusions d'ensemble ? En dépit du caractère très lacunaire de notre information, nous en présenterons quelques-unes, non sans les réserves nécessaires.

Il n'est sans doute pas très utile de revenir plus longuement sur les deux grandes phases qu'a connues le développement urbain dans le Nord de la Gaule. La seconde, qui correspond au Bas-Empire, est d'ailleurs la micux connue et présente un visage très uniforme; les problèmes en suspens concernent la date de la naissance de ces villes entourées de remparts et celle de leur disparition définitive, mais des éléments d'une chronologie plus précise pourront sans doute être réunis prochainement en France ${ }^{48}$.

(47) On peut naturellement aussi envisager une chronologie basse qui rejoindrait p. ex. le passage de Bononia a Cesoriacum - à condition que ce passage lui-mème ne date que de Caligula ou de Claude. Le nom d'Alugusia n'est plus considéré aujourd'hui comme une preuve de l'origgine angustéenne d'une fondation, cf. Kovtre, Trierer Zeilschr., 1938 , p. 192.

(48) Ces domnées seront fournies par l'étude des remparts des différents sites de la région. Nous avons fait 


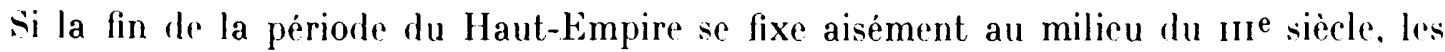
difficultés sont bien plus considérables pour les débuts. Différents stades doivent d'ailleurs ìtre envisagés. Il y a d'abord celui de la date de la fondation de la ville nouvelle ; car même si cette ville ne fait que perpétuer sous des formes rénovées un établissement gaulois - ce qui n'est pas, de loin. le cas le plus fréquent - l'urbaniste romain élabore un plan tout nouveau qui n'est réalisable que dans certaines conditions de terrain. Le plus souvent cependant l'emplacement choisi se trouve à quelque distance de l'ancienne agglomération. Ce transfert nous fournira une indication capitale : toutes ces villes nouvelles ont une place très précise dans le réseau routier que tisse soigneusement l'ingénieur romain. Nous avons rencontré ci-dessus une seule hésitation certaine ou, si l'on préfère, un seul changement de plan : celui qui concerne Vermand et Saint-Quentin-Augusta Viromanduorum, et encore s'agit-il là d'une ville qui ne fut sans doute jamais que de second plan ${ }^{49}$. Partout ailleurs, la solution initiale semble avoir été définitive, et elle n'a pu ètre qu'ancienne. Ia fondation - et. nous l'avons vu, il s'agit d'une fondation de toutes pièces - de cette plaque tournante d'importance capitale qu'est Bavay n'a-t-elle pas dù se placer tout à l'origine du plan d'organisation de la région? Mais une maille de ce réseau tient l'autre ${ }^{50}$ et on est amené de la sorte à dater ce plan d'organisation sous le règne d'Auguste et à le rattacher. conformément à la tradition, à son ministre déroué, Agrippa. De fait, les indices chronologiques que nous possédons pour Amiens et Bavay s'accordent avec ces déductions.

Fondation ne veut pas dire aménagement - c'est ce second point qui peut sembler d'une certaine manière plus important pour l'histoire du développement urbain. De fait. ce qui nous apparait sans doute avec le plus de netteté dans les fouilles. c'est l'état du ${ }_{\text {II }}$ e siècle et less vestiges qui dateraient des origines restent insaisissables. Pour l'instant. on ne peut poser de façon utile qu'une question, celle de l'existence et de l'adoption du plan à damier.

Il y a quelque apparence que ce plan ait été la règle pour les chefs-lieux de civitales et les agglomérations d'importance voisine. Il est sùr à Amiens, des plus probables à Bavay, probable ou possible à Boulogne. Mais il est pratiquement sùr aussi à Lutèce ${ }^{51}$, et il est bien connu dans les villes romaines d'Allemagne, de Suisse et d'Angleterre. A quelle date remontent ces plans à damier ? C’est une question à laquelle il est manifestement souvent difficile de répondre. Des précisions viennent d'Allemagne. Ine discussion serrée a été menée autour de Trèves et la thèse de H. Koethe qui se prononce pour une date claudienne parait en définitive la mieux fondée ${ }^{52}$. Pour Cologne, le quadrillage semble lié à la fondation de la colonie, c'est-à-dire vers l'an 50 de notre ère ${ }^{53}$. Ces deux villes importantes peuvent

comnajtre dans les études deja citèes celles conmues pour Amiens et Boulogne ; celles relatives à Fanars viennent d'ètre publiées dans cette revue par G. Bersu et W: Enverzagt (Gallia, XIX, 1961, 1, p. 158-190); el pour Bavay, il sera possible prochainement de presenter une mise au point.

(49) Ine seconde hésitation se manifeste - - peut-être - a Soissons ; du moins, la grande route venant du Nord se dirige sur l'oppidum de Pommiers, avant de faire un coude et de franchir l'Aisne.

(50) Naturellement ce misommement ne saurait valoir que pour les chefs-lieux de cicilates et autres villes importantes.

51 l.e fait semble ressortir du traci des roies connues et de l'orientation des idifices retrouvés, cf. P.-M. Duval, Paris anlique.

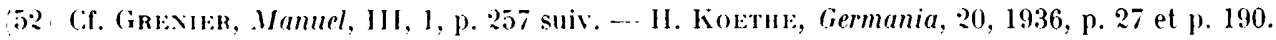

(53) C. les remarques d'ensemble d'll. v. Petrikovits, Las röm. Rheinland, p. k7 (avec hibliogr.). 
servir de points de repère pour une évolution qui a pu, au surplus, ètre graduelle. Pour le Nord de la Gaule, la seule donnée sùre est l'existence du plan à Amiens dès le ier siècle.

Ce plan à damier entrainait une délimitation de superficie : c'est un point sur lequel nous voyons un peu plus clair. Il convient naturellement de dire que cette superficie ne représentait qu'un programme; mais scules des fouilles exhaustives pourraient nous montrer si ces plans à damier furent construits et comment ils le furent. A Londres, nous dit-on, sur une surface totale de 130 ha intra muros. 73 seulement étaient sùrement prévus pour l'habitation; de mème, à Arenches le quartier sud-est ne semble jamais avoir étè bâti ${ }^{54}$. D'autre part, ces villes pouvaient posséder ou possédaient peut-être normalement leurs suburbia. De toute manière, ces superficies ne nous fournissent qu'un ordre de grandeur el il ne saurait guère ètre question d'éval uer d'après elles le chiffre de la population.

Comme ordre de grandeur, nous disposons d'abord du chiffre du plan définitif d'Amiens, soit une centaine d'hectares. Les points de comparaison ne manquent pas : 96 ha pour Cologne, 83 pour Xanten. 81 pour Trèves ; en Suisse. les chiffres sont un peu plus forts : 120 ha pour Augusta Raurica. 150 pour Avenches ; en Angleterre. on a 81 ha à Verulamium, 92 à Calleva-Silchester et 130 à Londres, chaque fois à l'intérieur des remparts du II $^{\mathrm{e}}$ siècle. Pour la Gaule chevelue, un seul chiffre directement comparable, celui d'Autun qui est encore d'une centaine d'hectares. Le cas de Lyon est malheureusement plus complexe : on compt: 62 ha pour la colonie, mais l'agglomération comportait deux autres quartiers ${ }^{55}$. Il appert de ces données que Samarobriva atteignit la position d'une grande ville à l'échelle de la région.

Il n'y a pas lieu de croire que ce fût là une moyenne. Nous arons pu faire état en effet. pour le voisinage immédiat, de deux autres chiffres. celui de Bavay avec une quarantaine d'hectares, celui de Boulogne, sans doute tres proche, ol il est somme toute assez probable que ce second chiffre devait ètre le plus fréquent, abstraction faite de certains candidat: a la centaine d'hectares, tols que Reims et Beaurais peut-ètre. En dehors de la région. on peut rapprocher les 48 ha de Canterbury-Duroternum et les 40 ha probables de la Colonia Julia Equestris (Nyon en Suisse).

Cie qui est notable, on l'a vu, c'est que Samarobriva fut fonclée avec un plan initial de 40 ha. seulement et, somme toute, sauf pour Trèves et Cologne, nous ne savons pas à quelle date remonte le plan à damier des autres villes citées. La centaine d'hectares peut aussi bien souvent correspondre à l'essor le plus grand atteint au ${ }_{11} \mathrm{e}$ siècle; ainsi il $\mathrm{nu}^{\circ}$ paraitra pas étonnant que la Colonia Clpia Traiana (Xanten) ait reçu une superficie de 83 ha. ou qu'Avenches, fondée sans doute sous les Flaviens, en reçut une de 1:0) ha. ${ }^{56}$.

Les chiffres que nous possédons semblent ainsi. en définitive, s'intégrer dans un tableau d'ensemble assez cohérent. (itons pour finir une donnée isolée qui nous renseignn' sur les agglomérations d'importance secondaire. Le Tournai du Haut-Empire semblr

(54) Le mème fait se reproduira d'ailleurs au Bas-limpire dans le cas de Treves.

(55) Pour l'Angleterre, cf. les remarques d'ensemble de 1. Richuoxo, Roman Brilain (Pelican IIislory of

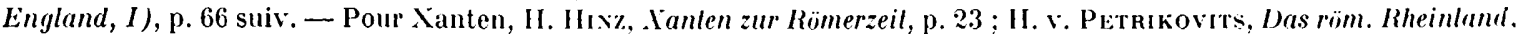
p. 96 suiv. -- Pour Lyon, Grexier, Manuel, III, 1, p. 220 et A. A. Drs, Essai sur la lopographie de Lughlumum. Pour Autun, superficie calculée d’après les deux dimensions N.-S. el E.-0. : 1.200 et 900 mètres environ.

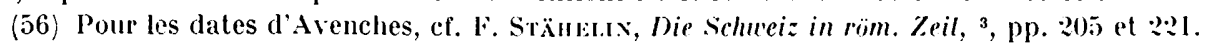


avoir atteint une dizaine d'hectares; ''e n'était qu'un gros vicus sous le Haut-Empire, mais qui se trouva bien plaré pour devenir chef-lieu de civitas sous le Bas-Empire, sans varier de superficie ${ }^{57}$.

In dernier point demande quelque commentaire : celui des remparts des villes du Haut-Empire. Le résultat des fouilles a été tout à fait négatif sur ce point; mais que vaut l'argumentum a silentio? On se le demandera avec d'autant plus de raisons que les murailles du Bas-Empire ont disparu le plus souvent sans laisser de traces. Mais à vrai dire. ce deuxième fait se comprend plus aisément, si l'on considère que les villes de cette époque se sont trouvées entièrement englobées dans les agglomérations médiévales et exposées à des atteintes continuelles. Ce sont précisément les enceintes du Haut-Empire qui se sont parfois mieux conservées, ainsi à Autun et à Avenches, mais il est peut-ètre assez normal que ces kilomètres de remparts aient laissé quelques vestiges et l'absence de tels vestiges peut être considérée comme significative ${ }^{58}$.

On peut s'étonner de cette apparente insouciance, alors que des villes situées plus loin de la frontière dangereuse comme Nimes, Orange, Lyon, Autun ou Avenches reçurent une ceinture fortifiée. Il se peut que ce soit là en partie un problème de chronologie; l'absence de remparts peut être la preuve du développement urbain relativement tardif de ces villes du Nord de la Gaule, développement qui peut se placer à un moment où la paix romaine et la sécurité des frontières paraissaient solidement assurées. Nul doute, en tout cas, que l'absence de fortifications leur fut fatale et causa la ruine de cette première civilisation urbaine que connut la région.

\section{Ernest WiLL.}

(57) M. Amaxd-I. liykexs-Dilerickx, Tournai romain (Disserlationes Gandenses, $\mathrm{v}$ ).

(58) Sur ce probleme des enceintes du debut de l'Empire, cf. en dernier lieu I. I Iarmand, Alli V II $^{\circ}$ Congresso 1rcheul. Classica, III. p. 195 ; à propos de Tongres, p. 198. Sur l'existence possible d'une enceinte de la même période a Tournai, cf. M. Amand-I. Erkexs-Dienickx, l. l., pp. 134-135. 